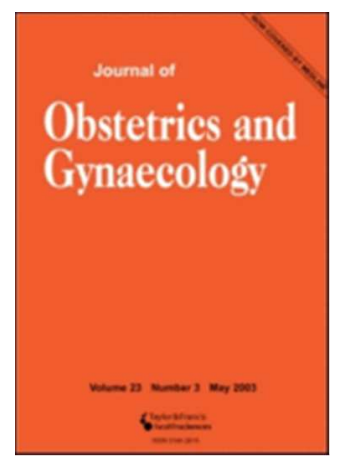

\title{
Combined laparoscopic excisional surgery for synchronous endometrial and rectal adenocarcinoma in an obese woman
}

\begin{tabular}{|c|c|}
\hline Journal: & Journal of Obstetrics and Gynaecology \\
\hline Manuscript ID & CJOG-2015-0843.R2 \\
\hline Manuscript Type: & Case Report \\
\hline Date Submitted by the Author: & 08-Aug-2016 \\
\hline Complete List of Authors: & $\begin{array}{l}\text { Macciò, Antonio; Azienda Ospedaliera Brotzu, Department of Gynaecologic } \\
\text { Oncology } \\
\text { Lavra, Fabrizio; Azienda Ospedaliera Brotzu, Department of Gynaecologic } \\
\text { Oncology } \\
\text { Chiappe, Giacomo; Azienda Ospedaliera Brotzu, Department of } \\
\text { Gynaecologic Oncology } \\
\text { Kotsonis, Paraskevas; Azienda Ospedaliera Brotzu, Department of } \\
\text { Gynaecologic Oncology } \\
\text { Sollai, Giuseppe; Azienda Ospedaliera Brotzu, Department of Oncological } \\
\text { Surgery } \\
\text { Zamboni, Fausto; Azienda Ospedaliera Brotzu, Department of General } \\
\text { Surgery } \\
\text { Madeddu, Clelia; University of Cagliari, Department of Medical Sciences } \\
\text { Mario Aresu }\end{array}$ \\
\hline Keywords: & $\begin{array}{l}\text { Gynaecological oncology, Gynaecological surgery, endometrial cancer, } \\
\text { rectal cancer, laparoscopy, obesity }\end{array}$ \\
\hline
\end{tabular}

\section{SCHOLARONE"}

Manuscripts 
Combined laparoscopic excisional surgery for synchronous endometrial and rectal adenocarcinoma in an obese woman

Antonio Macciò ${ }^{1}$, Fabrizio Lavra ${ }^{1}$, Giacomo Chiappe ${ }^{1}$, Paraskevas Kotsonis ${ }^{1}$, Giuseppe Sollai ${ }^{2}$, Fausto Zamboni ${ }^{3}$, Clelia Madeddu ${ }^{4}$

${ }^{1}$ Department of Gynaecologic Oncology, Azienda Ospedaliera Brotzu, Cagliari, Italy

${ }^{2}$ Department of Oncological Surgery, Azienda Ospedaliera Brotzu, Cagliari, Italy

${ }^{3}$ Department of General Surgery, Azienda Ospedaliera Brotzu, Cagliari, Italy

${ }^{4}$ Department of Medical Sciences "Mario Aresu", University of Cagliari, Italy

\section{Corresponding author:}

Antonio Macciò, MD

Department of Gynaecologic Oncology

A, Businco Hospital

Via Jenner Cagliari

Tel +390706095436

E-mail: a.maccio@tin.it

Keywords: endometrial cancer, rectal cancer, laparoscopy, obesity, 


\section{Introduction}

Endometrial cancer is the most common gynaecologic malignancy. Among all cancers, high body mass index (BMI) and obesity (defined as $\mathrm{BMI}>30 \mathrm{~kg} / \mathrm{m} 2$ ) are associated with the incidence and mortality rate of endometrial cancer (Reeves et al.,2007). In a metaanalysis of 19 reviews and prospective studies, Renehan et al. (2008) found that a $5 \mathrm{~kg} / \mathrm{m}^{2}$ increase in BMI significantly raised a woman's risk of developing endometrial cancer by a factor of 1.6. Colorectal cancer is also related to obesity and has been shown to be associated with endometrial cancers. Up to $10 \%$ of cases involving both, colorectal and endometrial cancers, may be caused by hereditary non-polyposis colorectal cancer (HNPCC), but the association is more frequently related to obesity.

An exhaustive review of the literature suggests that a minimally invasive approach has several recognised advantages over laparotomy with comparable oncological long-term results (Rabinovich, 2015; Bissolati et al., 2016), and to date it represents the surgical option of choice for both endometrial and rectal carcinoma. Therefore, when colorectal and endometrial cancers are simultaneously diagnosed, laparoscopic resection may be considered as an option. The present paper reports the case of an obese woman with synchronous endometrial and rectal cancers who underwent a same-sitting laparoscopic resection.

\section{Case Report}

A 78-year-old woman (BMI, $32 \mathrm{~kg} / \mathrm{m}^{2}$ ) was referred to the Department of Gynaecologic Oncology at A. Businco Hospital, Cagliari, for recurrent episodes of vaginal bleeding. The Department of Gynecologic Oncology is located in the A. Businco Hospital, which is the Regional Referral Center for Cancer diseases, and provides tertiary level of care for patients within the entire catchment area of the Sardinian Region, which include about $1,658,138$ resident people. The patient medical history was unremarkable. Transvaginal 
ultrasonography revealed hyperechoic endometrium approximately $3 \mathrm{~cm}$ thick. Endometrial cancer (G1) was diagnosed based on subsequent hysteroscopy with endometrial biopsy. Magnetic resonance imaging confirmed the presence of a uterine mass $(18 \times 28 \times 30 \mathrm{~mm})$ with suspected myometrial invasion involving more than $50 \%$ of the entire thickness of the uterine wall. Rectal wall thickening was also identified, and rectal cancer was suspected. Positron emission tomography/computed tomography (PET/CT) showed abnormally high uptake in the uterus and distal rectum. No abnormal uptake was identified at other locations. Additionally, no evidence of lymph node involvement was noted. Colonoscopy revealed an ulcerated mass causing stenosis in the rectum, which histological examination of a biopsy specimen identified as rectal adenocarcinoma. The case was discussed at a multidisciplinary team meeting for patients with suspected or confirmed malignancy and a simultaneous surgical approach for endometrial and rectal cancer was considered indicated.

\section{Surgical procedure}

The patient provided written informed consent for the surgical procedure after approval by the local institutional review board. The surgical team included Gynaecologists from the Department of Gynaecologic Oncology and Colorectal Surgeons from the Department of Oncological Surgery at the "Azienda Ospedaliera Brotzu", Cagliari.

Combined laparoscopic resection was performed under general anaesthesia. A 12-mm port was placed with an open technique in the umbilicus, and, in agreement with the team of anaesthesiologists to allow an effective Trendelenburg position, a pneumoperitoneum was established and maintained throughout the surgery by insufflating the peritoneal cavity with carbon dioxide $\left(\mathrm{CO}_{2}\right)$, at a pressure never exceeding $10 \mathrm{mmHg}$. Intra-abdominal visualization was achieved using a $10-\mathrm{mm}, 0^{\circ}$ telescope (Karl Storz, Tuttlingen, Germany). The technique involved placement of three additional trocars: a 12-mm trocar in the right 
lateral side, a 5-mm trocar in the suprapubic area between the umbilicus and pubic symphysis, and a 5-mm trocar in the left lateral side.

\section{Hysterectomy and bilateral salpingo-oophorectomy}

Following coagulation of the fallopian tubes, an intrauterine manipulator was used. The round ligaments were coagulated and divided using the LigaSure ${ }^{\mathrm{TM}}$ system (Covidien, Minneapolis, Minnesota, USA) to allow entry into the retroperitoneal space. The LigaSureTM system was used for all times of throughout the surgery. The ureter was visualized and isolated up to the uterus, and haemostatic clips were positioned bilaterally at the origins of the uterine arteries. Then, the infundibulopelvic ligament was coagulated and sectioned. An adequate margin of the vagina was ensured before performing colpectomy with a monopolar scissor. After completion of colpectomy, a Foley catheter was placed into the vagina to avoid pneumoperitoneum loss. The vaginal cuff was then closed laparoscopically using a continuous absorbable suture. The uterus and adnexa were extracted through the vagina.

\section{Low anterior rectal resection}

Following the avascular plane in front of Toldt's fascia and posterior to the mesocolon, the left mesocolon was completely mobilized to obtain a proximal colonic segment long enough for tension-free colorectal anastomosis at the pelvic floor level.

At the common iliac artery bifurcation, the medial mesenteric attachments were incised using a monopolar scissor, and the superior hypogastric nerve plexus was detected and retained. The pre-sacral space was entered, and total mesorectal excision was performed. Anterior traction on the rectum enabled sharp dissection in the rectosacral plane. Keeping the hypogastric nerves always in sight, the surgeon progressed inferiorly in a plane just anterior to the nerves. Dissection of the presacral space was accomplished from right to 
left and proceeded distally into the pelvis to the level of the levator muscles. Proximal lymph node dissection was performed at the origin of the superior rectal artery. The superior rectal artery was dissected following the application of the Ligasure. The inferior margin of the piriformis was used to identify the emerging sacral nerves. The same plane was developed laterally to the right and left, one side at a time. Dissection of the anterior rectum began by exposing the Denonvilliers fascia with identification of the vaginal wall. Dissection continued distally to expose the levator ani muscle. An Endo GIA $30 \mathrm{~mm}$ reload stapler (Covidien) was inserted through the right lower quadrant port and used to transect the rectum. Moving the rectum-sigma outside the pelvis, a bilateral pelvic and lombo-aortic lymphadenectomy was then performed up to the inferior mesenteric artery. The proximal colon was then extracted through a Pfannestiel incision of about $5 \mathrm{~cm}$. The excised rectum was exteriorized and examined for adequate margins. The frozen section of the rectal specimen showed clear surgical margins. The anvil of a size-29 circular stapler (Ethicon, Cincinnati, USA) was inserted and fixed to the colon with a size 0 polypropylene pursestring suture. The proximal colon was returned to the abdomen, followed by closure of the abdominal wall and re-establishment of the pneumoperitoneum. The circular stapler was then inserted into the rectum and carefully advanced to the apex of the rectal stump, and the anastomosis was closed. The anastomosis was tested by distending the rectum with air using a bulb syringe. After the fascial closure using 0 vicryl sutures with a fascia closure device, the skin was closed with 4-0 vicryl subcutaneous suture.

No vascular or visceral injury occurred during surgery. Additionally, no perioperative complications were noted. The estimated blood loss was $100 \mathrm{~mL}$, and the operation time was $400 \mathrm{~min}$. No wound hematoma, wound infection, or delayed bleeding was observed postoperatively. The patient was discharged from the hospital on postoperative day 9 in good condition. On definitive histological examination, the uterus showed a moderately differentiated endometrioid adenocarcinoma with squamocellular components infiltrating 
more than $50 \%$ of the myometrium (T1b), and the rectum showed a well-differentiated adenocarcinoma invading the rectal wall through the muscularis propria into the serosa (T3) (Figures 1 and 2). No metastasis was noted in the lymph nodes.

After obtaining consent from the patient and her family, formalin-fixed paraffin-embedded sections of the surgical specimens were examined immunohistochemically for abnormalities in the expression of MLH1 and MSH2 (DNA mismatch repair proteins). MLH1 and MSH2 expression was normal in both cancers. The patient was then referred to an Oncologist for adjuvant therapy followed by periodic follow-up that has included three PET/CT scan examinations to date. Thus far, at 24 months after initial surgery, the patient remains disease free.

\section{Discussion}

To the best of the authors' knowledge, the present paper is the first to describe a case of an obese woman with synchronous endometrial and rectal adenocarcinoma who underwent same-sitting laparoscopic resection of both cancers. Although the concomitance of these two tumours has been reported, their simultaneous diagnosis is rare. Only two cases of synchronous endometrial and rectal cancers, both occurring in patients with HNPCC, have been published (Sagawa et al., 2000; Komiyama et al., 2012). However, neither was treated laparoscopically. Notably, the choice to remove both tumours simultaneously may provide benefits both for the patient, in terms of improvement in quality of life, and for the surgeon, in terms of increased viability of the surgical plane, feasibility, and safety due to the decreased likelihood of adherences and related complications.

The combined laparoscopic surgery was technically feasible and safe, with no complications. The approach described here had an adequate operative time for endometrial and rectal cancers. Notably, the operating time of over 6 hours in the present 
case was shown to be safe and feasible. Indeed, a long operative time is not necessarily associated with increased postoperative complications (Harrison et al., 2014).

The limited blood loss in the present case is consistent with the median blood loss observed in large cohorts of laparoscopic hysterectomies (Macciò et al., 2016) and laparoscopic surgeries for colorectal cancer (van der Pas et al., 2013). Then, limited bleeding seems to be an achievable outcome.

Furthermore, the present case highlights the advantages of the laparoscopic approach for same-sitting surgery in women with synchronous endometrial and rectal cancers, even in those who are obese. Accordingly, the available data on the use of the laparoscopic approach in obese women with endometrial cancer (Eltabbakh et al., 2000) demonstrate that laparoscopic surgery is effective and safe, with a lower complication rate, shorter hospitalisation time, reduced postoperative pain, and better outcomes in terms of performance status and quality of life compared to laparotomy. Similarly, studies assessing the impact of obesity on laparoscopic surgery for rectal cancer found that BMI did not influence surgical morbidity, quality of surgery, or survival (Bissolati et al., 2016).

Therefore, the choice of a combined laparoscopic approach in an obese patient with synchronous endometrial and rectal cancers seems to be safe and feasible. Notably, however, such a complex surgical procedure requires a team with extensive expertise in laparoscopic oncological surgery.

\section{Acknowledgements}

This work supported by the "Associazione Sarda per la ricerca in Oncologia GinecologicaONLUS" with funding from the "Banco di Sardegna" Foundation (grant no. 5335, 2014). The authors thank Ivan Collu, Concetta di Simone, and Andreea Voicu for their technical assistance. 
Declaration of interest statement

The authors report no declarations of interest. 


\section{References}

Bissolati M, Orsenigo E, Staudacher C. 2016. Minimally invasive approach to colorectal cancer: an evidence-based analysis. Updates in Surgery 68: 37-46.

Eltabbakh GH, Shamonki MI, Moody JM, Garafano LL. 2000. Hysterectomy for obese women with endometrial cancer: laparoscopy or laparotomy? Gynecologic Oncology 78: 329-335.

Harrison OJ, Smart NJ, White P, Brigic A, Carlisle ER, Allison AS, Ockrim JB, Francis NK. 2014. Operative time and outcome of enhanced recovery after surgery after laparoscopic colorectal surgery. Journal of the Society of Laparoendoscopic Surgeons 18: 265-272.

Komiyama S, Nishio E, Ichikawa R, Miyamura H, Kawamura K, Komiyama M, Nishio Y, Udagawa Y. 2012. Asymptomatic synchronous quintuple primary cancers. Gynecologic and Obstetric Investigation 74: 324-328.

Macciò A, Chiappe G, Kotsonis P, Nieddu R, Lavra F, Serra M, Onnis P, Sollai G, Zamboni F, Madeddu C. 2016. Surgical outcome and complications of total laparoscopic hysterectomy for very large myomatous uteri in relation to uterine weight: a prospective study in a continuous series of 461 procedures. Archives of Gynecology and Obstetrics [Epub ahead of print]

Rabinovich A. 2015. Minimally invasive surgery for endometrial cancer: a comprehensive review. Archives of Gynecology and Obstetrics 291: 721-727.

Reeves GK, Pirie K, Beral V, Green J, Spencer E, Bull D. 2007. Cancer incidence and mortality in relation to body mass index in the Million Women Study: cohort study. British 
Medical Journal 335: 1134.

Renehan AG, Tyson M, Egger M, Heller RF, Zwahlen M. 2008. Body-mass index and incidence of cancer: a systematic review and meta-analysis of prospective observational studies. Lancet 371: 569-578.

Sagawa T, Yamada H, Yamamoto R, Sakuragi N, Fujimoto S. 2000. Two cases of endometrial cancer meeting new clinical criteria for hereditary nonpolyposis colorectal cancer. Gynecologic Oncology 79: 327-331.

van der Pas MH, Haglind E, Cuesta MA, Fürst A, Lacy AM, Hop WC, Bonjer HJ; COlorectal cancer Laparoscopic or Open Resection II (COLOR II) Study Group. 2013. Laparoscopic versus open surgery for rectal cancer (COLOR II): short-term outcomes of a randomised, phase 3 trial. Lancet Oncology 14: 210-218. 
Figure legends

Figure 1. Pathological specimen of rectal cancer. Dimension of the resected rectal cancer specimen.

Figure 2. Pathological specimen of rectal cancer. An ulcerated adenocarcinoma invading the rectal wall through the muscularis propria into the serosa (pT3) was found. 